Supporting Information for

\title{
Surface Chemistry Guides the Orientations of Adhering $E$. coli Cells Captured from Flow
}

Zhou Xu, ${ }^{1}$ Wuqi Amy Niu, ${ }^{2}$ Sylvia L. Rivera, ${ }^{3}$ Mark T. Tuominen, ${ }^{1}$ M. Sloan Siegrist,${ }^{3,4}$ and Maria M. Santore ${ }^{2,4^{*}}$

1. Department of Physics, University of Massachusetts, Amherst, MA 01003

2. Department of Polymer Science and Engineering, University of Massachusetts Amherst, MA 01003

3. Department of Microbiology, University of Massachusetts Amherst, MA 01003

4. Molecular and Cellular Biology Graduate Program, University of Massachusetts, Amherst, MA 01003

*corresponding author: Maria Santore

Department of Polymer Science and Engineering

University of Massachusetts

120 Governors Drive

Amherst, MA 01003

413-577-1417

santore@mail.pse.umass.edu

\section{Shear Force and Torque on an Adherent E. coli Cell Treated as a Rod}

\section{Velocity profile}

The velocity near the flow chamber wall, $\mathrm{U}$, is linear in distance from the wall, $\mathrm{y}$, and proportional to the wall shear rate, $\gamma$ :

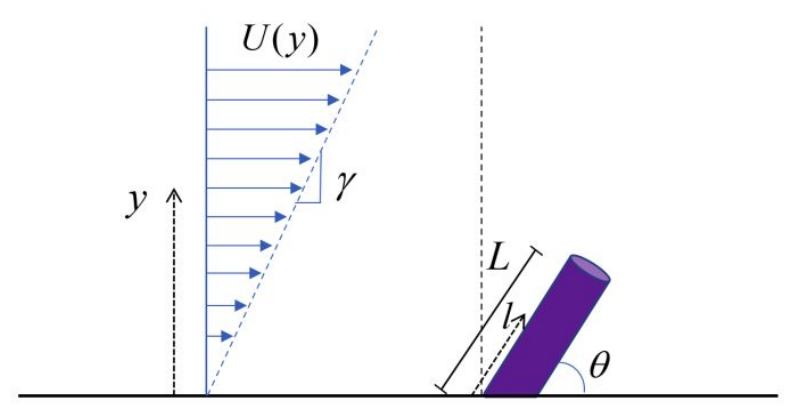

Figure SI 1. Figure 7 of the main manuscript reproduced here for necessssity. Diagram showing geometry and variables for force and torque calculations. 
$l$ measures the distance along the bacterium from the adhered point at the surface to its free end at $l=L$. The cell is tilted at angle $\theta$ from the surface, such that the velocity can be expressed at any point $l$ on the cell.

$$
U(l)=\mu l \sin \theta
$$

\section{Calculating the Torque on a cell}

The cell is treated as a tilted rod of radius $r$. A differential slice, taken parallel to the flow chamber surface, in Figure SI 2 will have an elliptical cross section, having an aspect ratio

$$
\frac{a}{b}=\frac{1}{\sin \theta}
$$

Where $\boldsymbol{a}$ is the long axis and $\boldsymbol{b}$ is the short axis. (Note that the cell itself has a circular cross section, but slicing a tilted cell parallel to the wall produces an ellipse shape.)

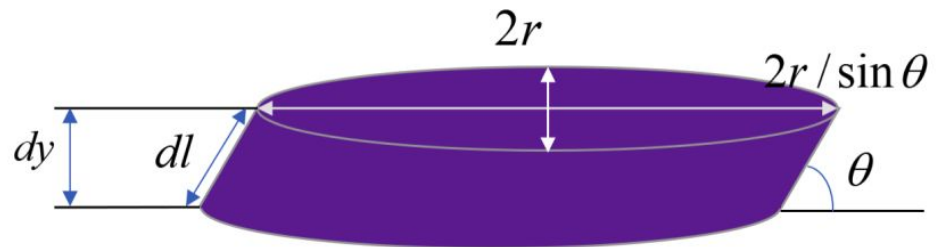

Figure SI 2. A differential slice of a cell, sliced parallel to the surface and showing the elliptical cross-cut.

For the elliptical differential element at small Reynolds number (low flow rate), the drag coefficient for cross flow can be approximated as [1] 
and

$$
\begin{aligned}
& C_{D}=\frac{4 \pi}{R_{e} S} \\
& S=\frac{1}{2}(1+\sigma)-c-\ln \frac{R_{e}}{8(1+\sigma)} .
\end{aligned}
$$

Where $\sigma=(a-b) /(a+b)$ and $c=0.57721 \ldots$ is Euler's constant. $R_{e}$ is the particle Reynolds number, defined as

$$
R_{e}=\frac{2 r U \rho}{\mu}
$$

where $\rho$ and $\mu$ are the fluid density and viscosities, respectively.

The drag force follows a defined relationship with the drag coefficient,

$$
F_{D}=\frac{1}{2} \rho U^{2} C_{D} A
$$

where $A$ is cross area projected in the flow direction.

For a differential slice of the elliptical cylinder, the projected area is $2 r \sin \theta d l$. Substituting this into the expression for drag force, a differential drag force, $d F_{D}$, on a differential section of the cell results,

$$
d F_{D}=\frac{1}{2} C_{D} \rho U^{2}(2 r) \sin \theta d l
$$

The differential torque, $d \tau$, on the differential element follows

$$
d \tau=C_{D} \rho r U^{2} \sin ^{2} \theta l d l .
$$

It is useful to express $l$ as a function of $R_{e}$ by combining equations (2) and (5): 


$$
l=\frac{\mu}{2 r \rho \gamma \sin \theta} R_{e} .
$$

Using equation (9), the integration in equation (8) $d l$ can be simplified to produce an integration with a single dimensionless integration variable $R_{e}$, noting that $S$ is a function of $R_{e}$,

$$
\tau=\frac{\pi \mu^{4}}{4 r^{3} \rho^{3} \gamma^{2}} \int_{0}^{R_{e}(L)} \frac{R_{e}{ }^{2}}{S} d R_{e}
$$

Here $R_{e}(L)$ is the Reynolds number at outer tip of the $E$. coli cell. For $\gamma=5 \mathrm{~s}^{-1}$, cell length $L=3 \mu \mathrm{m}$ and $r=0.5 \mu m, R_{e}(L)=1.5 \times 10^{-5} \sin \theta$.

Numerical integration yields the normalized torque on the cell, with respect to that for a vertical cell, as a function of tilt angle in Figure SI 3.

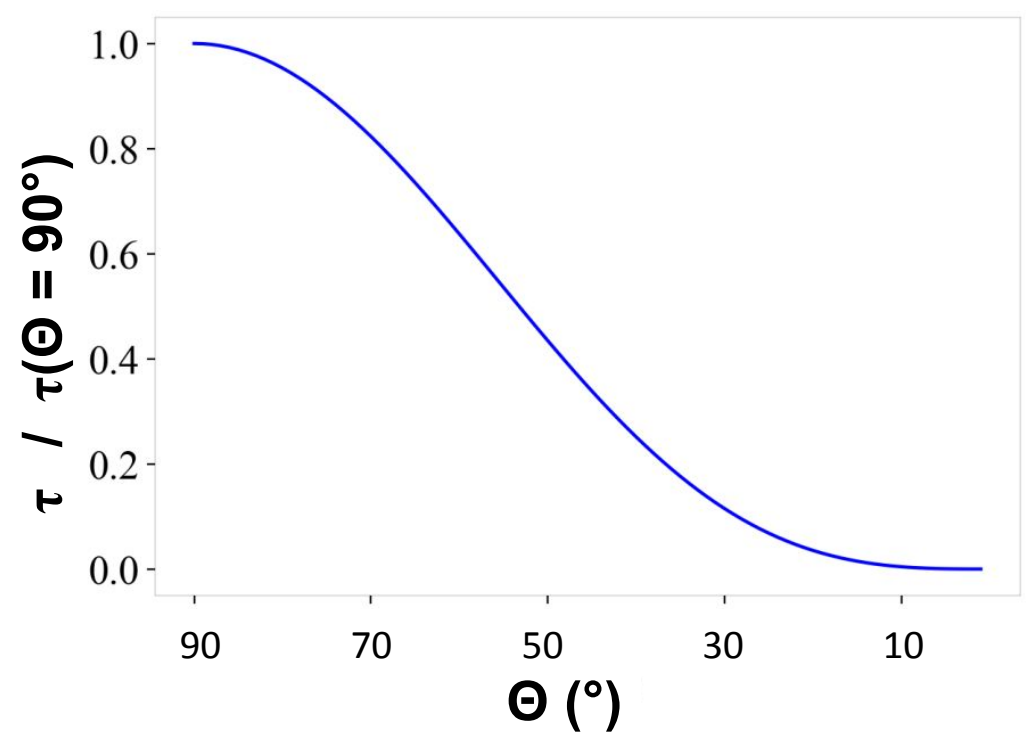

Figure SI 3. The torque on a cell as a function of tilt angle $\theta$, normalized on the torque on a vertical cell. 
[1] S. Tomotika, T. Aoi, "The Steady Flow of a Viscous Fluid Past an Elliptic Cylinder at Small Reynolds Numbers" 